Article

\title{
Effect of Dihydroartemisinin-Piperaquine on the Pharmacokinetics of Praziquantel for Treatment of Schistosoma mansoni Infection
}

\author{
Omary Mashiku Minzi ${ }^{1}$, Rajabu Hussein Mnkugwe ${ }^{2,3}$ (D) $^{\text {Eliford Ngaimisi }}{ }^{4}$ (D), Safari Kinung'hi ${ }^{5}$, \\ Anna Hansson ${ }^{6}$, Anton Pohanka ${ }^{2,6}$, Appolinary Kamuhabwa ${ }^{1}$ and Eleni Aklillu ${ }^{2, *(D)}$
}

1 Department of Clinical Pharmacy and Pharmacology, School of Pharmacy, Muhimbili University of Health and Allied Sciences, 11103 Dar es Salaam, Tanzania; minziobejayesu@gmail.com (O.M.M.); enali2012@gmail.com (A.K.)

2 Division of Clinical Pharmacology, Department of Laboratory Medicine, Karolinska Institutet, 14186 Stockholm, Sweden; rajabuhussein06@gmail.com (R.H.M.); anton.pohanka@sll.se (A.P.)

3 Department of Clinical Pharmacology, School of Medicine, Muhimbili University of Health and Allied Sciences, 11103 Dar es Salaam, Tanzania

4 Office of Clinical Pharmacology, Division of Pharmacometrics, Food and Drugs Administration, Silver Spring, MD 20993, USA; engaimisi@gmail.com

5 National Institute for Medical Research, Mwanza Research Centre, 33104 Mwanza, Tanzania; kinunghi_csm@hotmail.com

6 Department of Clinical Pharmacology, Karolinska University Hospital-Huddinge, 14186 Stockholm, Sweden; anna.b.hansson@sll.se

check for updates

Citation: Minzi, O.M.; Mnkugwe, R.H.; Ngaimisi, E.; Kinung'hi, S.; Hansson, A.; Pohanka, A.;

Kamuhabwa, A.; Aklillu, E. Effect of Dihydroartemisinin-Piperaquine on the Pharmacokinetics of Praziquantel for Treatment of Schistosoma mansoni Infection. Pharmaceuticals 2021, 14, 400. https://doi.org/10.3390/ ph14050400

Academic Editor: Conor R. Caffrey

Received: 28 March 2021

Accepted: 21 April 2021

Published: 23 April 2021

Publisher's Note: MDPI stays neutral with regard to jurisdictional claims in published maps and institutional affiliations.

Copyright: (c) 2021 by the authors. Licensee MDPI, Basel, Switzerland. This article is an open access article distributed under the terms and conditions of the Creative Commons Attribution (CC BY) license (https:// creativecommons.org/licenses/by/ $4.0 /)$.

* Correspondence: eleni.aklillu@ki.se; Tel.: +46-73-511-6131

\begin{abstract}
Praziquantel (PZQ) and dihydroartemisinin-piperaquine (DHP) combination recently showed superior effectiveness than PZQ alone to treat intestinal schistosomiasis. In this follow-up study, we investigated the effect of DHP co-administration on the pharmacokinetics of PZQ and its enantiomers among 64 Schistosoma mansoni infected children treated with PZQ alone $(n=32)$ or PZQ + DHP combination $(n=32)$. Plasma samples collected at $0,1,2,4,6$, and $8 \mathrm{~h}$ post-dose were quantified using UPLCMS/MS. The geometric mean (GM) of AUCs for total PZQ, R-PZQ and S-PZQ were significantly higher among children who received PZQ + DHP than PZQ alone. The geometric mean ratio (GMR) and $(90 \% \mathrm{CI})$ of $\mathrm{AUC}_{0-\infty}$ for PZQ + DHP to PZQ for total PZQ, R-PZQ, and S-PZQ were $2.18(1.27,3.76), 3.98(2.27,7.0)$ and $1.86(1.06,3.28)$, respectively. The GMR and $(90 \% \mathrm{CI})$ of $\mathrm{AUC}_{0-8}$ for total PZQ, R-PZQ, and S-PZQ were $1.73(1.12,2.69), 2.94(1.75,4.92)$, and $1.50(0.97,2.31)$, respectively. The GM of $C_{\max }$ for total PZQ, R-PZQ and S-PZQ were significantly higher among those who received PZQ + DHP than PZQ alone. The GMR $(90 \% \mathrm{CI})$ of $\mathrm{C}_{\max }$ of PZQ + DHP to PZQ for total PZQ, R-PZQ, and S-PZQ were $1.75(1.15,2.65), 3.08(1.91,4.96)$, and 1.50 $(1.0,2.25 \%)$, respectively. The $90 \%$ CI of the GMRs for both AUCs and $C_{\max }$ for total PZQ, R-PZQ, and S-PZQ were outside the acceptable 0.80-1.25 range, indicating that the two treatment arms were not bioequivalent. DHP co-administration significantly increases systemic PZQ exposure, and this may contribute to increased effectiveness of PZQ + DHP combination therapy than PZQ alone to treat schistosomiasis.
\end{abstract}

Keywords: dihydroartemisinin-piperaquine; praziquantel; praziquantel enantiomers pharmacokinetics interaction; Schistosoma mansoni

\section{Introduction}

Schistosomiasis is a poverty-related parasitic infection that prevails in rural and disadvantaged populations in the tropics and sub-tropics regions [1,2]. It is estimated that more than 250 million people are infected with schistosomiasis globally [3,4]. A large proportion of the global burden is from children of Sub-Saharan Africa (SSA) [5,6]. Schistosomiasis is associated with anemia, poor growth, impaired physical fitness, and poor cognitive abilities 
in affected children [7-10] and delay in the achievement of Sustainable Development Goals (SDGs) [11,12].

Mass drug administration (MDA) using praziquantel (PZQ) targeting school-aged children is a World Health Organization (WHO)-recommended global strategy to control schistosomiasis in endemic settings [13]. Despite the use of mass PZQ treatment for several years, schistosomiasis remains a public health problem in most endemic countries in SSA, including Tanzania $[8,14,15]$. The lack of PZQ activity against the immature schistosome (schistosomula) is suggested as one of the reasons for the failure of MDA with PZQ in eliminating the disease as a public health problem [16]. Furthermore, an alarming threat of PZQ resistance in field studies from SSA has been previously reported [17]. Therefore, there is pressing need for a broad anthelminthic approach supplementing PZQ with new antischistosomal drugs that targets different parasite developmental stages and/or with a different mechanism of action [18], to increase treatment efficacy and reduce the risk of PZQ resistance [19].

Combination of drugs with different mechanisms of action has become a common strategy to increase efficacy and reduce or delay the development of drug resistance. Previous studies indicated that artemisinin derivatives have capacity to kill immature schistosomes hence improving the efficacy of PZQ [16,20,21]. In a randomized clinical trial, we recently reported superior effectiveness of PZQ plus dihydroartemisinin-piperaquine (DHP) combination therapy targeting to kill both mature and immature parasites than PZQ alone for the treatment of schistosomiasis in infected children [22]. As the use of combination of drugs increases, the likelihood of drug interactions, any effect of DHP on the pharmacokinetics of PZQ and its enantiomers needs to be investigated. Most pharmacokinetic drug interactions occur at a metabolic level altering drug metabolism and disposition. Dihydroartemisinin (DHA), an artemisinin derivative, is mainly eliminated via glucuronidation by UDP-glucuronosyltransferases (UGTs) UGT1A9 and UGT2B7 [23]. Both PZQ and piperaquine (PPQ, an aminoquinoline derivative) are metabolized by cytochrome P450 (CYP450) enzymes mainly via CYP3A4 and this may pose a risk of drug-drug interactions [24,25]. Chloroquine (CHQ), which is also an aminoquinoline derivative like PPQ [26] is reported to reduce the bioavailability and maximum concentration $\left(C_{\max }\right)$ of PZQ in humans despite the non-competitive inhibition on PZQ metabolism [27]. Therefore, the likelihood of drug-drug interactions of clinical relevance between PZQ and DHP when co-administered for treatment of schistosomiasis warrant further investigation.

PZQ is a racemic mixture of R-praziquantel (R-PZQ) and S-praziquantel (S-PZQ) enantiomers, and recent findings reported higher antischistosomal activity of R-PZQ enantiomer when administered alone compared to the racemate at the same dose [28,29]. Overall data on the pharmacokinetics profile of PZQ and its enantiomers among infected children is scarce, and any potential drug interactions of PZQ and DHP combination therapy albeit increased efficacy, remains to be investigated [22]. This study compared the pharmacokinetic profiles of PZQ and its enantiomers when PZQ is given alone or in combination with DHP for treatment of Schistosoma mansoni infection among school children in North-Western Tanzania.

\section{Results}

\subsection{Sociodemographic and Baseline Characteristics}

A total of 64 Schistosoma mansoni infected children (32 received PZQ alone and 32 received PZQ + DHP) were enrolled and completed this study. The mean age $( \pm \mathrm{SD})$ of the study population was $12.7 \pm 1.8$ years. Females were $45.3 \%(29 / 64)$ of all study participants. There was no significant difference in patient's and baseline characteristics between treatment arms $(p>0.05)$ (Table 1$)$. 
Table 1. Sociodemographic and baseline characteristics of study participants.

\begin{tabular}{|c|c|c|c|c|}
\hline \multirow{2}{*}{\multicolumn{2}{|c|}{ Variable }} & \multicolumn{2}{|c|}{ Treatment Arm } & \multirow{2}{*}{$p$-Value } \\
\hline & & \multirow{2}{*}{$\begin{array}{c}\text { PZQ } \\
12.8 \pm 1.6 \\
10-17\end{array}$} & \multirow{2}{*}{$\begin{array}{c}\text { PZQ + DHP } \\
12.7 \pm 2.0 \\
9-16\end{array}$} & \\
\hline Age (years) & $\begin{array}{c}\text { Mean } \pm \mathrm{SD} \\
\text { Range }\end{array}$ & & & $0.79^{\mathrm{a}}$ \\
\hline \multirow{2}{*}{ Sex } & Male N (\%) & $17(53.1)$ & $18(56.3)$ & 0.85 \\
\hline & Female N (\%) & $15(46.9)$ & $14(43.8)$ & 0.87 \\
\hline Weight (kg) & Mean \pm SD & $33.1 \pm 6.2$ & $33.7 \pm 9.3$ & $0.74^{\mathrm{a}}$ \\
\hline Height $(\mathrm{cm})$ & Mean \pm SD & $141.0 \pm 9.7$ & $143.9 \pm 12.9$ & $0.32^{\mathrm{a}}$ \\
\hline Haemoglobin (g/dL) & Mean \pm SD & $12.4 \pm 1.7$ & $12.2 \pm 1.9$ & $0.71^{\mathrm{a}}$ \\
\hline \multirow{4}{*}{ Infection intensity } & Eggs per gran Median (range) & $246(12-1452)$ & $165(6-1722)$ & $0.32^{b}$ \\
\hline & Light N (\%) & $9(28.1)$ & $14(43.8)$ & 0.46 \\
\hline & Moderate N (\%) & $11(34.4)$ & $8(25.0)$ & 0.67 \\
\hline & Heavy N (\%) & $12(37.5)$ & $10(31.3)$ & 0.77 \\
\hline
\end{tabular}

a_-Independent Student $t$-test; ${ }^{\mathrm{b}}$-Manny Whitney U test; SD—Standard deviation.

\subsection{Effect of DHP on the Pharmacokinetic Parameters of Total PZQ}

The arithmetic and geometric means of $\mathrm{AUC}_{0-8}$ and $\mathrm{AUC}_{0-\infty}$ for total PZQ were significantly higher among children who received PZQ + DHP combination than those who received PZQ alone (Table 2). The final linear mixed effect (LME) models included treatment arm $(p=0.028)$ and Body Mass Index (BMI) $(p=0.042)$ as statistically significant predictors of total PZQ $C_{\max }$; treatment arm $(p=0.041)$ and BMI $(p=0.021)$ as statistically significant predictors of total PZQ AUC ${ }_{0-8} ;$ treatment arm $(p=0.021)$ and BMI $(p=0.079)$ as predictors of total PZQ AUC $0-\infty$; and treatment arm $(p=0.007)$ and sex $(p=0.012)$ as statistically significant predictors of total PZQ half-life.

Table 2. Comparisons of pharmacokinetic parameters of total PZQ between PZQ + DHP and PZQ treatment arms.

\begin{tabular}{cccc}
\hline \multirow{2}{*}{ Parameters } & \multicolumn{2}{c}{ Treatment Arm } & (PZQ + DHP) I PZQ \\
\cline { 2 - 4 } & Geometric mean $(\mathrm{CV} \%)$ & Geometric mean $(\mathrm{CV} \%)$ & Geometric Mean Ratio (90\% CI) \\
\hline AUC $(0-\mathrm{Inf})$ & $66.8(112.1)$ & $33.5(106.4)$ & $2.18(1.27-3.76)$ \\
\hline AUC $(0-8 \mathrm{~h})$ & $50.4(181.8)$ & $32.4(111.9)$ & $1.73(1.12-2.69)$ \\
\hline $\mathrm{C}_{\max }(\mathrm{ng} / \mathrm{mL})$ & $14.3(169)$ & $8.9(95.1)$ & $1.75(1.15-2.65)$ \\
\hline Half-life $(\mathrm{h})$ & $1.5(29.7)$ & $2.2(47.5)$ & $0.7(0.57-0.86)$ \\
\hline $\mathrm{T}_{\max }(\mathrm{h})$ & $3.6(55.7)$ & $3.5(61.8)$ & \\
\hline
\end{tabular}

The $\mathrm{AUC}_{0-\infty}$ geometric mean ratio (GMR) of PZQ + DHP to PZQ for total PZQ was 2.18 and $90 \% \mathrm{CI}$ of $1.27-3.76$. For $\mathrm{AUC}_{0-8}$, the GMR was 1.73 and $90 \% \mathrm{CI}$ of $1.12-2.69$. The $90 \%$ CI of GMRs for both $\mathrm{AUC}_{0-\infty}$ and $\mathrm{AUC}_{0-8}$ of total PZQ were outside the bioequivalence limits of 0.80-1.25. Infected children who received PZQ + DHP combination had significantly higher total PZQ systemic exposure than those who received PZQ alone. Comparison of the mean dose-normalized concentration-time profile and box plot of $\mathrm{AUC}_{0-8}$ for total PZQ in the presence and absence of DHP is presented in Figures 1 and 2, respectively. The arithmetic and geometric means of $C_{\max }$ of total PZQ were significantly higher among children who received PZQ + DHP combination than those who received PZQ alone. The GMR of PZQ + DHP to PZQ of the $C_{\max }$ was 1.75 and $90 \%$ CI of 1.15-2.65 (Table 2). The observed $90 \% \mathrm{CI}$ of the GMR for $\mathrm{C}_{\max }$ for total PZQ was also outside the acceptable range 
of 0.80-1.25. There was no significant effect of DHP on the elimination half-life (hours) and time to reach maximum plasma concentration ( $T_{\max }$ in hours) of total PZQ (Table 2). The arithmetic means $( \pm \mathrm{SD})$ and medians (IQR) of the pharmacokinetics parameters for total PZQ are presented in the supplementary file (Table S1).
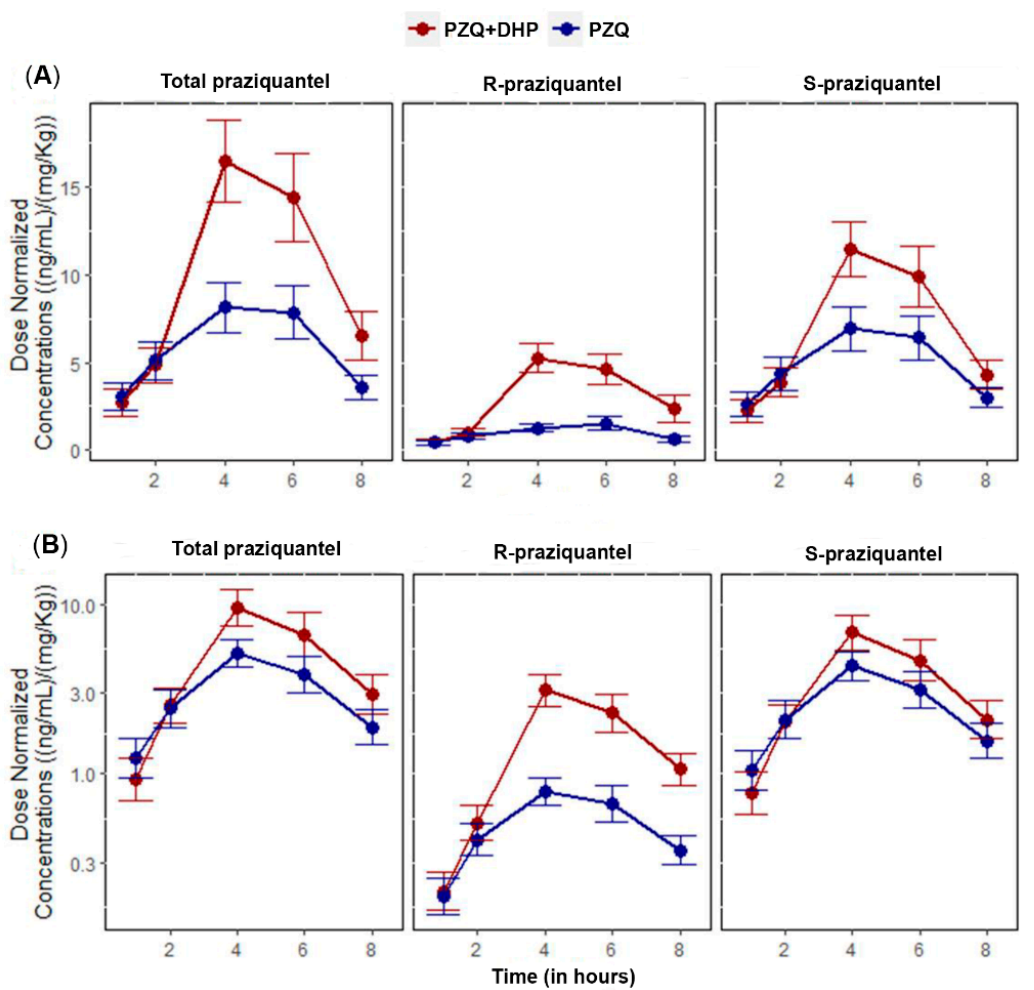

Figure 1. Comparisons of mean of dose-normalized concentration-time profiles of total PZQ, R-PZQ and S-PZQ between PZQ versus PZQ + DHP treatment arms. Error bars represent standard errors of the mean (SEM). (A) Linear/linear scale (B) Log/linear scale.

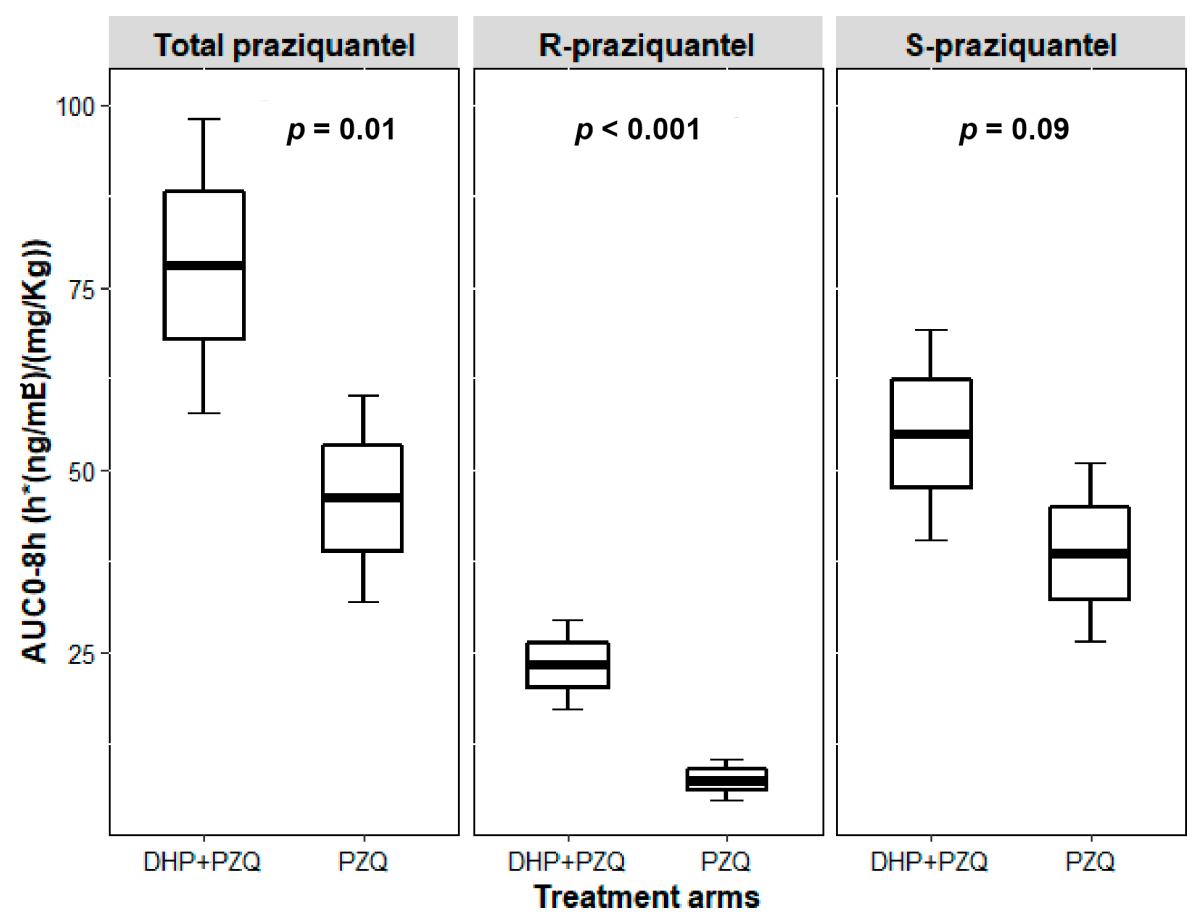

Figure 2. Boxplots comparing dose-normalized $\mathrm{AUC}_{0-8 \mathrm{~h}}$ for total PZQ, R-PZQ and S-PZQ between PZQ versus PZQ + $\mathrm{DHP}$ treatment arms. Boxes represent mean $\pm \mathrm{SE}$, error bars represent mean $\pm 1.96^{*} \mathrm{SE}$. 


\subsection{Effect of DHP on the Pharmacokinetic Parameters of R-PZQ and S-PZQ}

Like what was observed with total PZQ, the arithmetic and geometric means of $\mathrm{AUC}_{0-8}$ and $\mathrm{AUC}_{0-\infty}$ for R-PZQ and S-PZQ were significantly higher among children who received PZQ + DHP combination than those who received PZQ alone (Table 3). The arithmetic and geometric means of the AUCs and $\mathrm{C}_{\max }$ of S-PZQ were higher than the one for R-PZQ (Table 3).

Table 3. Comparisons of pharmacokinetic parameters of R-PZQ and S-PZQ between PZQ + DHP and PZQ treatment arms.

\begin{tabular}{|c|c|c|c|c|c|c|}
\hline \multirow[b]{3}{*}{ Parameters } & \multicolumn{2}{|c|}{ R-PZQ } & \multirow[b]{3}{*}{$\begin{array}{c}(P Z Q+D H P) \mid P Z Q \\
\text { GMR }(90 \% \text { CI })\end{array}$} & \multicolumn{2}{|c|}{ S-PZQ } & \multirow[b]{3}{*}{$\begin{array}{c}(\mathrm{PZQ}+\mathrm{DHP}) \mid \mathrm{PZQ} \\
\text { GMR }(90 \% \mathrm{CI})\end{array}$} \\
\hline & \multicolumn{2}{|c|}{ Treatment Arm } & & \multicolumn{2}{|c|}{ Treatment Arm } & \\
\hline & $\begin{array}{c}\text { PZQ + DHP } \\
\text { GM (CV\%) }\end{array}$ & $\begin{array}{c}\text { PZQ } \\
\text { GM }(C V \%)\end{array}$ & & $\begin{array}{l}\text { PZQ + DHP } \\
\text { GM (CV\%) }\end{array}$ & $\begin{array}{c}\text { PZQ } \\
\text { GM }(\mathrm{CV} \%)\end{array}$ & \\
\hline AUC (0-Inf) & $\begin{array}{c}19.5 \\
(118.1)\end{array}$ & $\begin{array}{c}4.9 \\
(84.2)\end{array}$ & $3.98(2.27-7.0)$ & $\begin{array}{c}44.8 \\
(114.6)\end{array}$ & $\begin{array}{c}27.1 \\
(119.6)\end{array}$ & $1.86(1.06-3.28)$ \\
\hline AUC (0-8h) & $\begin{array}{c}14.2 \\
(235.3)\end{array}$ & $\begin{array}{c}4.8 \\
(144.1)\end{array}$ & $2.94(1.75-4.92)$ & $\begin{array}{c}35.6 \\
(174.9)\end{array}$ & $\begin{array}{c}26.7 \\
(114.9)\end{array}$ & $1.5(0.97-2.31)$ \\
\hline $\mathrm{C}_{\max }(\mathrm{ng} / \mathrm{mL})$ & $\begin{array}{c}4.5 \\
(206.5)\end{array}$ & $\begin{array}{c}1.5 \\
(119.5)\end{array}$ & $3.08(1.91-4.96)$ & $\begin{array}{c}9.9 \\
(160.1)\end{array}$ & $\begin{array}{c}7.3 \\
(96.3)\end{array}$ & $1.5(1.0-2.25)$ \\
\hline Half-life (h) & $\begin{array}{c}1.6 \\
(34.3)\end{array}$ & $\begin{array}{c}2 \\
(35.9)\end{array}$ & $0.79(0.63-0.98)$ & $\begin{array}{c}1.7 \\
(26.7)\end{array}$ & $\begin{array}{c}2.1 \\
(43.5)\end{array}$ & $0.77(0.63-0.94)$ \\
\hline $\mathrm{T}_{\max }(\mathrm{h})$ & $\begin{array}{c}3.8 \\
(51.4)\end{array}$ & $\begin{array}{c}3.5 \\
(62.8)\end{array}$ & & $\begin{array}{c}3.6 \\
(55.2)\end{array}$ & $\begin{array}{c}3.5 \\
(61.8)\end{array}$ & \\
\hline
\end{tabular}

$\mathrm{CV}-$ Coefficient of variation; GM-Geometric mean.

The final LME models for R-PZQ included treatment arm $(p<0.001)$ only as a statistically significant predictor of R-PZQ $\mathrm{C}_{\max }$; treatment arm $(p<0.001)$ only as a statistically significant predictor of R-PZQ AUC ${ }_{0-8}$; treatment arm $(p<0.001)$ only as statistically significant predictor of R-PZQ $\mathrm{AUC}_{0-\infty}$; and treatment arm $(p=0.074)$ as predictors of R-PZQ half-life. The final LME models for S-PZQ included treatment arm $(p=0.102)$ and BMI $(p=0.022)$ as significant predictors of S-PZQ $C_{\max }$; treatment arm $(p=0.123)$ and BMI $(p=0.009)$ as predictors of S-PZQ AUC $_{0-8} ;$ treatment $\operatorname{arm}(p=0.072)$ and BMI $(p=0.052)$ as predictors of S-PZQ AUC $\mathrm{AU}_{0}$; and treatment $\operatorname{arm}(p=0.035)$ and $\operatorname{sex}(p=0.047)$ as predictors of S-PZQ half-life.

For R-PZQ, the GMR of PZQ + DHP to PZQ for $\mathrm{AUC}_{0-\infty}$ was 3.98 and $90 \% \mathrm{CI}$ of 2.27-7.00. For $\mathrm{AUC}_{0-8}$, the GMR was 2.94 and $90 \% \mathrm{CI}$ of $1.75-4.92$. The $90 \% \mathrm{CI}$ of the GMRs for $\mathrm{AUC}_{0-\infty}$ and $\mathrm{AUC}_{0-8}$ were outside bioequivalence limits of $0.80-1.25$. The arithmetic and geometric means of $C_{\max }$ for R-PZQ were significantly higher among children who received $\mathrm{PZQ}+\mathrm{DHP}$ combination than those who received PZQ alone. The GMR of PZQ + DHP to PZQ of the $C_{\max }$ for R-PZQ was 3.08 and $90 \%$ CI of $1.91-4.96$ (Table 3). The $90 \%$ CI of the GMR for $C_{\max }$ like AUCs was also outside the bioequivalence limits of 0.80-1.25.

For S-PZQ, the GMR of PZQ + DHP to PZQ for $\mathrm{AUC}_{0-\infty}$ was 1.86 and $90 \% \mathrm{CI}$ of 1.06-3.28. For $\mathrm{AUC}_{0-8}$, the GMR was 1.50 and $90 \% \mathrm{CI}$ of $0.97-2.31$. Similar to R-PZQ, the $90 \% \mathrm{CI}$ of the GMRs for both $\mathrm{AUC}_{0-\infty}$ and $\mathrm{AUC}_{0-8}$ for S-PZQ were outside the bioequivalence limits of $0.80-1.25$. The arithmetic and geometric means of $C_{\max }$ for $S-P Z Q$ were higher among children who received PZQ + DHP combination than those who received PZQ alone. The GMR of PZQ + DHP to PZQ of the $C_{\max }$ for S-PZQ was 1.50 and $90 \%$ CI of 1.0-2.25 (Table 3). The $90 \%$ CI of the GMR for $C_{\max }$ was also outside the bioequivalence limits of 0.80-1.25. Comparison of mean dose-normalized concentration-time profile and box plot of $\mathrm{AUC}_{0-8}$ for R-PZQ and S-PZQ in the presence and absence of DHP are presented in Figures 1 and 2, respectively. There was no significant effect of DHP on elimination half-life (hours) and $\mathrm{T}_{\max }$ in hours of both R-PZQ and S-PZQ (Table 3). The arithmetic means $( \pm S D)$ and medians (IQR) of the pharmacokinetics parameters for R-PZQ and S-PZQ are presented in the supplementary file (Tables S2 and S3). 


\section{Discussion}

In a randomized clinical trial, we recently reported that PZQ and DHP combination therapy is safe and more efficacious than PZQ alone for the treatment of intestinal schistosomiasis in infected children [22]. Drugs co-administered with PZQ may have a similar CYP450 metabolic pathway and potentially alter PZQ systemic exposure, which may in turn influence treatment outcomes. This follow-up pharmacokinetic drug-interaction study investigated the effect of DHP on the pharmacokinetic profile of total PZQ and its enantiomers among Schistosoma mansoni infected school children. Our results indicate a significant drug-drug interaction between DHP and PZQ, in which systemic drug exposure increased among infected children treated with PZQ + DHP combination therapy than those who received PZQ monotherapy as reflected by the increased $A U C_{s}$ and $C_{\max }$. The change in AUC of a drug, representing its systemic exposure, is the main parameter considered in the evaluation of drug-drug interactions (DDIs) [30]. To our knowledge, this is the first study to investigate drug interaction between DHP and PZQ and its clinical relevance in Schistosoma mansoni infected children.

Our result indicates significantly higher $\mathrm{AUC}_{0-\infty}, \mathrm{AUC}_{0-8}$ and $\mathrm{C}_{\max }$ of both total PZQ and its enantiomers R-PZQ and S-PZQ among children treated with PZQ + DHP combination therapy than those who received PZQ alone. The $90 \% \mathrm{CI}$ of the geometric mean ratios of AUCs and $C_{\max }$ for both total PZQ, R-PZQ, and S-PZQ were outside the acceptable bioequivalent interval of $0.80-1.25$ (Tables 2 and 3), indicating that systemic exposure of PZQ in the presence and absence of DHP is not bioequivalent.

In the combination therapy arm, participants received PZQ with a fixed dose dihydroartemisinin and piperaquine combination (DHP). Dihydroartemisinin is eliminated via glucuronidation catalyzed by UDP-glucuronosyltransferases, mainly by UGT1A9 and UGT2B7 [23]. Both PZQ and piperaquine undergo metabolism via cytochrome P450 enzymes, primarily by CYP3A4 [24,25]. Based on their metabolic pathways, the observed significant increase in plasma PZQ exposure is most likely due to competitive inhibition of CYP3A4/ 5 enzymes by piperaquine but not by dihydroartemisinin. CYP3A-mediated drug interaction is a possible mechanism by which DHP co-administration increases systemic PZQ exposure as reflected by significantly high $\mathrm{AUC}_{0-\infty}, \mathrm{AUC}_{0-8}$, and $\mathrm{C}_{\max }$ for total PZQ and its enantiomers among children who received PZQ + DHP combination therapy. A similar inhibition effect on PZQ metabolism by another aminoquinoline drug chloroquine is reported previously $[26,27]$.

Using a randomized clinical trial, we recently reported a significantly higher cure rate both at 3- and 8-weeks post-treatment in Schistosoma mansoni infected children who were treated with PZQ + DHP combination therapy compared to PZQ alone [22]. This follow-up drug-interaction study reveals that co-administration of DHP significantly increases plasma exposure and bioavailability of PZQ. In pharmacokinetics-pharmacodynamics (PK-PD) studies, high plasma drug concentration is used as a surrogate for a high drug concentration in tissue. Accordingly, higher plasma PZQ systemic exposure by DHP co-administration can enhance PZQ therapeutic efficacy and increased the cure rate. Indeed, PZQ is reported to show dose-dependent treatment response in school-aged children, increasing cure rates and egg reduction rates with escalating dosages of PZQ [31]. A previous PZQ PK-PD study reported that higher total PZQ AUC sere associated with an increased parasitological cure, and a higher PZQ dose is required for maximal efficacy [32]. PZQ + DHP combination therapy is advantageous not only for killing both mature and immature stages of the parasite [18] and protection against malaria [33] but also in increasing systemic PZQ exposure and its therapeutic efficacy. Thus, the reported higher efficacy among the children treated with PZQ + DHP combination therapy could possibly be due to both higher systemic PZQ exposure and the killing of immature schistosomes by DHP.

PZQ administration has two major drawbacks, the first being the high dose needed ( $40 \mathrm{mg} / \mathrm{kg}$ body weight) and the second is its bitter and unpleasant taste [34]. Younger children usually cannot swallow the existing tablets $(600 \mathrm{mg})$ because of their large size and bitter taste, causing compliance problems. Apart from increasing efficacy, the ob- 
served significant DDIs in the PZQ + DHP combination therapy can possibly alleviate the drawbacks of PZQ monotherapy. Our result indicates that the inhibitory effect of DHP is more pronounced for R-PZQ than the S-PZQ (Figure 2), and this can be an advantage for the combination therapy since R-PZQ is the therapeutically active enantiomer and has less bitter taste compared to racemic PZQ [34]. Therefore PZQ-DHP combination therapy not only offers the chance to reduce PZQ dose, but also the potential to reduce the pill burden and the unpleasant taste of the racemic PZQ. Synergistic and additive drug-drug interactions with the intention to reduce the dose of PZQ while still having an efficacious effect using the antimalarial mefloquine was explored in rodents previously [35]. A similar approach can be investigated for PZQ-DHP combination in humans.

Higher PZQ systemic drug exposure may result in not only increased effectiveness but also occurrence of adverse events among those treated with PZQ + DHP combination therapy. However, no significant difference in the overall occurrence of adverse events was reported between children treated with PZQ + DHP combination and PZQ alone [22]. Therefore, an increased systemic PZQ exposure by co-administration of DHP without affecting safety, yet improved efficacy, is an important finding which complements the current standard treatment and can serve as an alternative regimen to PZQ monotherapy.

\section{Materials and Methods}

\subsection{Study Design, Area, and Population}

This was a parallel two-arm pharmacokinetic study to investigate potential pharmacokinetic drug interaction between PZQ and DHP and its clinical relevance. This study was part of a randomized clinical trial registered with PACTR201612001914353 [22]. A total of 64 Schistosoma mansoni infected school children aged 9-17 years old attending Fogofogo Primary School in Busega district, Simiyu region, North-Western Tanzania who participated in our previous randomized clinical trial [22] were included in this follow-up pharmacokinetic drug-interaction study. The study area was a rural village located along the shores of Lake Victoria endemic for intestinal schistosomiasis.

\subsection{Treatment and Samples Collection for Pharmacokinetic Study}

Pre-treatment standardized meal was given to study participants as reported previously [22,36], as food has been known to increase PZQ bioavailability [37,38]. A total of 64 Schistosoma mansoni infected children were treated with either single-dose PZQ $40 \mathrm{mg} / \mathrm{kg}$ bodyweight $(n=32)$ alone or PZQ + DHP combination therapy $(n=32)$. The dose of DHP tablets (40 mg dihydroartemisinin / 320mg piperaquine) was calculated based on the child's body weight (in $\mathrm{kg}$ ) and was given once per day for three consecutive days following the WHO treatment guideline for uncomplicated malaria [39]. PZQ and the first dose of DHP were administered at zero hour. Two $\mathrm{mL}$ of venous blood was collected at 0 , $1,2,4,6$ and $8 \mathrm{~h}$ post-dose from the antecubital arm vein using an indwelling intravenous catheter into heparinized vacutainer tubes. Blood samples were centrifuged at $1000 \mathrm{rpm}$ for $10 \mathrm{~min}$ to obtain plasma stored at $-80^{\circ} \mathrm{C}$ freezer for drug quantification.

\subsection{Chemicals and Reagents}

R-PZQ, S-PZQ and rac-PZQ were purchased from Merck (Darmstadt, Germany). An eleven-fold rac-deuterated-PZQ (rac-PZQ-d11) as an internal standard (IS) was purchased from Toronto Research Chemicals (Toronto, Ontario, Canada). Figure 3 presents the chemical structure of R-PZQ and S-PZQ [38]. Acetone, acetonitrile, ammonium acetate, isopropanol, methanol, and acetic acid of mass spectrometry (MS) grade were purchased from Merck (Darmstadt, Germany). Deionized water/Milli-Q water/ultrapure water was prepared using a Milli-Q water purification system (Merck Millipore, MA, USA). Blank human plasma was kindly supplied by the blood bank of the Karolinska University Hospital Huddinge (Stockholm, Sweden). 


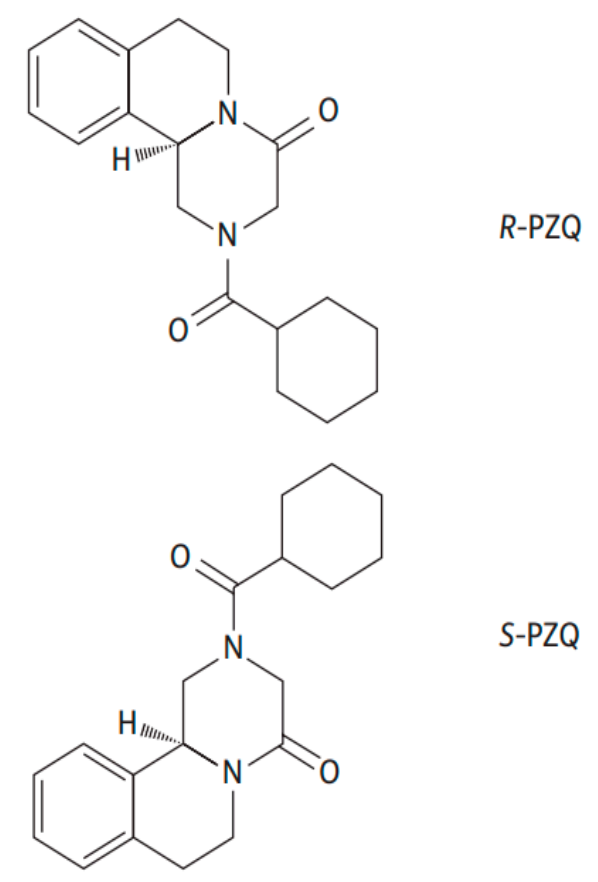

Figure 3. The chemical structure of R-PZQ and S-PZQ enantiomers [38].

\subsection{Analytical Method and Validation}

A two-channel system consisting of a Dionex Ultimate 3000RS liquid chromatography (LC) system with a TriPlus RSI autosampler and a Thermo Scientific TSQ Quantis triple quadrupole mass spectrometer (MS/MS) was used to quantify R-PZQ and S-PZQ. The MS/MS was operated in positive ionization mode. The data were processed using TraceFinder 4.1 (Thermo Scientific). The LC-MS/MS method for analysis of R-PZQ and S-PZQ was adapted from a recently validated enantioselective method described by Kovac et al. [28]. Briefly, plasma calibration samples were freshly prepared by spiking blank plasma samples with rac-PZQ and were included in each analytical run. Quality control samples were also prepared by spiking plasma blanks to obtain low, medium, and high concentrations for both R-PZQ and S-PZQ. The quantification range of the method was 1 to $1500 \mathrm{ng} / \mathrm{mL}$ for both R-PZQ and S-PZQ.

For extraction of analytes of interest, $100 \mu \mathrm{L}$ of plasma samples went through protein precipitation with $200 \mu \mathrm{L}$ of internal standard solution $(50 \mathrm{ng} / \mathrm{mL}$ of rac-PZQ-d11 in methanol) and then vortexed for $10 \mathrm{~s}$ followed by centrifugation for $5 \mathrm{~min}$ at $2100 \times g$. $150 \mu \mathrm{L}$ of the supernatant was diluted with $75 \mu \mathrm{L}$ Milli-Q water and $5 \mu \mathrm{L}$ was injected onto the LC-MS/MS system. The chromatographic system was using a Chiralpak AGP $2.0 \times 100 \mathrm{~mm}, 5 \mu \mathrm{m}$, column (Chiral Technologies Europe, Illkirch, France) with $10 \mathrm{mM}$ ammonium acetate: isopropanol 98:2 $(v / v)$ pH 8 as mobile phase with a flow rate of $0.3 \mathrm{~mL} / \mathrm{min}$. The chromatographic run was $22 \mathrm{~min}$, and with use of the parallel twochannel capacity, injection to injection time was $11 \mathrm{~min}$. R-PZQ eluted first followed by S-PZQ with a difference of $1.9 \mathrm{~min}$.

Praziquantel was monitored by the transition $\mathrm{m} / \mathrm{z} 313.2>202.9$ and the IS rac-PZQd11by $324.2>204.1$. The analytical method was validated according to the European Medicines Agency Guideline on bioanalytical method validation [40]. The calibration curve was constructed by linear regression of the analyte/internal standard area ratios with an applied weighing of $1 / x^{2}$. Accuracy was within $\pm 5 \%$ except for lower limit of quantification (LLOQ) where it was within $\pm 12 \%$. Precision was below $5 \mathrm{CV} \%$ except for LLOQ (below $13 \mathrm{CV} \%$ ). 


\subsection{Pharmacokinetic Data Analysis}

Noncompartmental analysis (NCA) with linear trapezoidal rule was used to calculate pharmacokinetics parameters using $\mathrm{R}$ statistical software version 4.0.2 [41]. The primary and secondary pharmacokinetic parameters include maximum plasma concentration $\left(\mathrm{C}_{\max }\right)$ in $\mathrm{ng} / \mathrm{mL}$, time needed to reach $\mathrm{C}_{\max }\left(\mathrm{T}_{\max }\right)$ in hours, area under the concentration-time curve from zero hour to infinity $\left(\mathrm{AUC}_{0-\infty}, \mathrm{ng} \cdot \mathrm{h} / \mathrm{mL}\right)$, area under the concentration-time curve from zero hour to $8 \mathrm{~h}$ post-dose $\left(\mathrm{AUC}_{0-8 \mathrm{~h}}, \mathrm{ng} \cdot \mathrm{h} / \mathrm{mL}\right)$ and terminal half-life $\left(\mathrm{t}_{1 / 2}\right)$ in hours for R-PZQ, S-PZQ, and total PZQ. $C_{\max }, \mathrm{T}_{\max }, \mathrm{t}_{1 / 2}, \mathrm{AUC}_{0-8 \mathrm{~h}}$, and $\mathrm{AUC}_{0-\infty}$ were calculated from the pharmacokinetics raw data using the PKNCA package version 0.9.4 implemented in R [42].

\subsection{Statistical Data Analysis}

Summary statistics of the pharmacokinetic parameters for each treatment group were reported as arithmetic mean, standard deviation (SD), geometric mean and coefficient of variation $(\mathrm{CV} \%)$. The geometric mean is a mean or average which indicates the central tendency of the data that have been log 10-transformed before statistical analysis (e.g., AUC or $C_{\max }$ ) and calculated as antilogarithm of the mean of the $\log 10$ transformed data. Coefficient of variation (CV \%) was calculated using the formula CV\% $=\sqrt{\mathrm{e}^{\text {Variance of } X}-1}$ where $\mathrm{X}$ is log-transformed pharmacokinetic parameter values. Concentrations, AUCs and $C_{\max }$ were dose-normalized by dividing concentration or pharmacokinetics parameter values by received dose. Independent $t$-test was used to compare the means of the logtransformed $\mathrm{AUC}_{0-8}$ between treatment groups.

Linear mixed effect modeling was used to determine subject-characteristics that influence noncompartmental pharmacokinetic parameters values $\left(\mathrm{C}_{\max }, \mathrm{AUC}_{0-8}, \mathrm{AUC}_{0-\infty}\right.$, and $t_{1 / 2}$ ) of R-PZQ, S-PZQ, and total PZQ. The evaluated subject-characteristics for each pharmacokinetic parameter included age, weight, BMI, treatment arm (PZQ + DHP vs PZQ) and sex. For each pharmacokinetic parameter, a model of log-transformed values versus treatment arm was built as a base model. Additional subject-characteristics were added individually to the base model in a stepwise manner. An added subjectcharacteristic was retained in the model if it was found to be significantly associated with the pharmacokinetic parameter $(p<0.05)$. The final modes were parameterized as follows: $\ln (\mathrm{PK}) \sim \beta \times \mathrm{TRT}+\alpha_{\mathrm{i}} \times \mathrm{CHAR}_{\mathrm{i}}+\varepsilon$, where PK represents pharmacokinetic parameters, $\beta$ represents fixed effect parameter for the treatment arm (PZQ being the reference arm), $\alpha_{\mathrm{i}}$ represents fixed effect parameter for additional subject-characteristics $\left(\mathrm{CHAR}_{\mathrm{i}}\right)$, and $\varepsilon$ represents between-subject variability of the pharmacokinetics. Parameters of the final models were used to calculate the geometric mean ratio (GMR) of the test (PZQ + DHP) to reference (PZQ) treatment arms and corresponding $90 \%$ confidence intervals (CI). In brief, GMR was calculated as antilogarithm of $\beta$, while $90 \%$ CI were calculated using the final model $\varepsilon$ in the test arm, $\beta$, degrees of freedom in the test arm $(n=28)$ and alpha value of 0.05 . The $90 \%$ confidence interval (CI) of the GMRs for AUCs and $C_{\max }$ were calculated to assess bioequivalence between PZQ + DHP versus PZQ alone treatment. Drug-drug interaction was concluded when the $90 \%$ CI of GMR of PZQ + DHP to PZQ ratio for a pharmacokinetic parameter was not entirely contained within the acceptable bioequivalence limits of $0.80-1.25$ (no effect boundaries).

\section{Conclusions}

In summary, DHP co-administration significantly increases systemic exposure of total PZQ and its enantiomers. Our study provides a new insight in which CYP3Amediated drug interaction as a possible contributor for the increased effectiveness of the PZQ + DHP combination therapy than PZQ monotherapy for the treatment and control of schistosomiasis. DHP+PZQ combination therapy is beneficial not only to kill both mature and immature stages of the parasites but also increases PZQ bioavailability and its therapeutic efficacy. The synergistic and additive drug-drug interactions between DHP and PZQ without compromising safety makes DHP+PZQ combination therapy as 
a better alternative than PZQ alone for MDA in schistosomiasis control and elimination program. However, any effect of PZQ co-administration on DHP pharmacokinetics needs further investigation.

Supplementary Materials: The following are available online at https:/ /www.mdpi.com/article/10 $.3390 / \mathrm{ph} 14050400 / \mathrm{s} 1$, Table S1: Comparisons of pharmacokinetic parameters of total PZQ between PZQ + DHP and PZQ treatment arms, Table S2: Comparisons of pharmacokinetic parameters of R-PZQ between PZQ + DHP and PZQ treatment arms, Table S3: Comparisons of pharmacokinetic parameters of S-PZQ between PZQ + DHP and PZQ treatment arms.

Author Contributions: Conceptualization, O.M.M., R.H.M., A.K., and E.A.; methodology, O.M.M., R.H.M., S.K., A.K., and E.A.; software, S.K., A.P., A.H., E.N., E.A.; validation, O.M.M., R.H.M., A.P., A.H., A.K., and E.A.; formal analysis, O.M.M., R.H.M., E.N., S.K., A.P., A.H., A.K., and E.A.; investigation, O.M.M., R.H.M., A.H., A.P., S.K., A.K., and E.A.; resources, O.M.M., E.N., A.H., A.P., S.K., A.K., and E.A.; data curation, O.M.M., R.H.M., E.N., S.K., A.P., A.H., A.K., and E.A.; writingoriginal draft preparation, O.M.M., and R.H.M.; writing-review and editing, O.M.M., R.H.M., E.N., S.K., A.P., A.H., A.K., and E.A.; visualization, O.M.M., R.H.M., E.N., S.K., A.K., and E.A.; supervision, O.M.M., S.K., A.K., and E.A.; project administration, O.M.M., A.K., and E.A.; funding acquisition, O.M.M., A.K., and E.A. All authors have read and agreed to the published version of the manuscript.

Funding: This study was supported by Sida-MUHAS capacity building grant through its bilateral program between Sweden and Tanzania (grant number 51170072).

Institutional Review Board Statement: The study was conducted according to the guidelines of the Declaration of Helsinki, and approved by the Medical Research Coordination Committee (MRCC) of the National Institute for Medical Research (NIMR Head Quarters), Dar es Salaam, Tanzania (Ref. No. NIMR/HQ/R.8a/Vol.IX/2343) (7 November 2016), Muhimbili University of Health and Allied Sciences Institutional Review Board (Ref. No. 2016-5-25/AEC/Vol.X/03) (25 May 2016) and Stockholm Ethics Committee (Ref. No. 2020-00845) (7 April 2020).

Informed Consent Statement: Before enrollment of the participants, information about the study was given and written informed consent from parents/guardians and assent from participating school children were obtained.

Data Availability Statement: All data presented in this study are contained within the manuscript and its supporting file.

Acknowledgments: We wish to thank our study participants including parents, guardians and children for their participation in this study. We acknowledge the technical support from staff at NIMR Mwanza Research Centre during recruitment of study participants and data collection. We thank the Fogofogo Primary School administration and community leaders for logistical support and guidance during data collection.

Conflicts of Interest: The authors declare no conflict of interest.

\section{References}

1. Chitsulo, L.; Engels, D.; Montresor, A.; Savioli, L. The global status of schistosomiasis and its control. Acta Trop. 2000, 77, 41-51. [CrossRef]

2. Hotez, P.J.; Alvarado, M.; Basanez, M.G.; Bolliger, I.; Bourne, R.; Boussinesq, M.; Brooker, S.J.; Brown, A.S.; Buckle, G.; Budke, C.M.; et al. The global burden of disease study 2010: Interpretation and implications for the neglected tropical diseases. PLoS Negl. Trop. Dis. 2014, 8, e2865. [CrossRef] [PubMed]

3. World Health Organization. Schistosomiasis Fact Sheet. Available online: http://www.who.int/mediacentre/factsheets/fs115 /en/index.html (accessed on 13 February 2020).

4. Mazigo, H.D. Participatory integrated control strategies and elimination of schistosomiasis in sub-Saharan Africa. Lancet Glob. Health 2019, 7, e998-e999. [CrossRef]

5. World Health Organization. Neglected Tropical Diseases, Hidden Successes, Emerging Opportunities; World Health Organization: Geneva, Switzerland, 2009; Available online: https:/ /apps.who.int/iris/handle/10665/44214 (accessed on 15 February 2020).

6. World Health Organization. Schistosomiasis and Soiltransmitted Helminthiases: Numbers of People Treated in 2017; World Health Organization: Geneva, Switzerland, 2018; Available online: https:/ /www.who.int/neglected_diseases/resources/who_wer9350/ en/ (accessed on 30 January 2020). 
7. Frigerio, S.; Macario, M.; Iacovone, G.; Dussey-Comlavi, K.J.; Narcisi, P.; Ndiaye, A.T.; Moramarco, S.; Alvaro, R.; Palombi, L.; Buonomo, E. Schistosoma haematobium infection, health and nutritional status in school-age children in a rural setting in Northern Senegal. Minerva Pediatr. 2016, 68, 282-287.

8. Kinung'hi, S.M.; Mazigo, H.D.; Dunne, D.W.; Kepha, S.; Kaatano, G.; Kishamawe, C.; Ndokeji, S.; Angelo, T.; Nuwaha, F. Coinfection of intestinal schistosomiasis and malaria and association with haemoglobin levels and nutritional status in school children in Mara region, Northwestern Tanzania: A cross-sectional exploratory study. BMC Res. Notes 2017, 10, 583. [CrossRef]

9. Koukounari, A.; Fenwick, A.; Whawell, S.; Kabatereine, N.B.; Kazibwe, F.; Tukahebwa, E.M.; Stothard, J.R.; Donnelly, C.A.; Webster, J.P. Morbidity indicators of Schistosoma mansoni: Relationship between infection and anemia in Ugandan schoolchildren before and after praziquantel and albendazole chemotherapy. Am. J. Trop. Med. Hyg. 2006, 75, 278-286. [CrossRef]

10. Stoltzfus, R.J.; Albonico, M.; Tielsch, J.M.; Chwaya, H.M.; Savioli, L. Linear growth retardation in Zanzibari school children. J. Nutr. 1997, 127, 1099-1105. [CrossRef]

11. Hotez, P.J.; Fenwick, A.; Savioli, L.; Molyneux, D.H. Rescuing the bottom billion through control of neglected tropical diseases. Lancet 2009, 373, 1570-1575. [CrossRef]

12. Hotez, P.J.; Herricks, J.R. Helminth elimination in the pursuit of sustainable development goals: A "worm index" for human development. PLoS Negl. Trop. Dis. 2015, 9, e0003618. [CrossRef]

13. World Health Organization. Investing to Overcome the Global Impact of Neglected Tropical Diseases, Third WHO Report on Neglected Tropical Diseases; World Health Organization: Geneva, Switzerland, 2015; Available online: https://apps.who.int/iris/bitstream/ handle/10665/152781/9789241564861_eng.pdf?sequence=1 (accessed on 6 November 2019).

14. Mnkugwe, R.H.; Minzi, O.S.; Kinung'hi, S.M.; Kamuhabwa, A.A.; Aklillu, E. Prevalence and correlates of intestinal schistosomiasis infection among school-aged children in North-Western Tanzania. PLoS ONE 2020, 15, e228770. [CrossRef]

15. Munisi, D.Z.; Buza, J.; Mpolya, E.A.; Kinung'hi, S.M. Schistosoma mansoni infections, undernutrition and anaemia among primary schoolchildren in two onshore villages in Rorya district, North-Western Tanzania. PLoS ONE 2016, 11, e0167122. [CrossRef]

16. Inobaya, M.T.; Olveda, R.M.; Chau, T.N.; Olveda, D.U.; Ross, A.G. Prevention and control of schistosomiasis: A current perspective. Res. Rep. Trop. Med. 2014, 2014, 65-75. [CrossRef]

17. Gryseels, B.; Mbaye, A.; De Vlas, S.J.; Stelma, F.F.; Guisse, F.; Van Lieshout, L.; Faye, D.; Diop, M.; Ly, A.; Tchuem-Tchuente, L.A.; et al. Are poor responses to praziquantel for the treatment of Schistosoma mansoni infections in Senegal due to resistance? An overview of the evidence. Trop. Med. Int. Health 2001, 6, 864-873. [CrossRef]

18. Bergquist, R.; Elmorshedy, H. Artemether and Praziquantel: Origin, Mode of Action, Impact, and Suggested Application for Effective Control of Human Schistosomiasis. Trop. Med. Infect. Dis. 2018, 3, 125. [CrossRef]

19. World Health Organization. Prevention and Control of Schistosomiasis and Soil-Transmitted Helminthiasis: Report of a WHO Expert Committee; Technical Report Series 912; World Health Organization: Geneva, Switzerland, 2002; Available online: https://apps. who.int/iris/handle/10665/42588 (accessed on 6 November 2019).

20. Liu, R.; Dong, H.F.; Guo, Y.; Zhao, Q.P.; Jiang, M.S. Efficacy of praziquantel and artemisinin derivatives for the treatment and prevention of human schistosomiasis: A systematic review and meta-analysis. Parasites Vectors 2011, 4, 201. [CrossRef]

21. Perez del Villar, L.; Burguillo, F.J.; Lopez-Aban, J.; Muro, A. Systematic review and meta-analysis of artemisinin based therapies for the treatment and prevention of schistosomiasis. PLOS ONE 2012, 7, e45867. [CrossRef]

22. Mnkugwe, R.H.; Minzi, O.; Kinung'hi, S.; Kamuhabwa, A.; Aklillu, E. Efficacy and safety of praziquantel and dihydroartemisinin piperaquine combination for treatment and control of intestinal schistosomiasis: A randomized, non-inferiority clinical trial. PLoS Negl. Trop. Dis. 2020, 14, e0008619. [CrossRef]

23. Ilett, K.F.; Ethell, B.T.; Maggs, J.L.; Davis, T.M.; Batty, K.T.; Burchell, B.; Binh, T.Q.; Thu Le, T.A.; Hung, N.C.; Pirmohamed, M.; et al. Glucuronidation of dihydroartemisinin in vivo and by human liver microsomes and expressed UDP-glucuronosyltransferases. Drug Metab. Dispos. 2002, 30, 1005-1012. [CrossRef] [PubMed]

24. Lee, T.M.; Huang, L.; Johnson, M.K.; Lizak, P.; Kroetz, D.; Aweeka, F.; Parikh, S. In vitro metabolism of piperaquine is primarily mediated by CYP3A4. Xenobiotica 2012, 42, 1088-1095. [CrossRef] [PubMed]

25. Wang, H.; Fang, Z.Z.; Zheng, Y.; Zhou, K.; Hu, C.; Krausz, K.W.; Sun, D.; Idle, J.R.; Gonzalez, F.J. Metabolic profiling of praziquantel enantiomers. Biochem. Pharmacol. 2014, 90, 166-178. [CrossRef] [PubMed]

26. Karunajeewa, H.A.; Ilett, K.F.; Mueller, I.; Siba, P.; Law, I.; Page-Sharp, M.; Lin, E.; Lammey, J.; Batty, K.T.; Davis, T.M. Pharmacokinetics and efficacy of piperaquine and chloroquine in Melanesian children with uncomplicated malaria. Antimicrob. Agents Chemother. 2008, 52, 237-243. [CrossRef] [PubMed]

27. Masimirembwa, C.M.; Naik, Y.S.; Hasler, J.A. The effect of chloroquine on the pharmacokinetics and metabolism of praziquantel in rats and in humans. Biopharm. Drug Dispos. 1994, 15, 33-43. [CrossRef] [PubMed]

28. Kovac, J.; Meister, I.; Neodo, A.; Panic, G.; Coulibaly, J.T.; Falcoz, C.; Keiser, J. Pharmacokinetics of praziquantel in Schistosoma mansoni- and Schistosoma haematobium-infected school- and preschool-aged children. Antimicrob. Agents Chemother. 2018, 62, e02253-17. [CrossRef]

29. Meister, I.; Ingram-Sieber, K.; Cowan, N.; Todd, M.; Robertson, M.N.; Meli, C.; Patra, M.; Gasser, G.; Keiser, J. Activity of praziquantel enantiomers and main metabolites against Schistosoma mansoni. Antimicrob. Agents Chemother. 2014, 58, 5466-5472. [CrossRef] [PubMed]

30. Choi, Y.H. Interpretation of drug interaction using systemic and local tissue exposure changes. Pharmaceutics 2020, $12,417$. [CrossRef] 
31. Coulibaly, J.T.; Panic, G.; Silue, K.D.; Kovac, J.; Hattendorf, J.; Keiser, J. Efficacy and safety of praziquantel in preschool-aged and school-aged children infected with Schistosoma mansoni: A randomised controlled, parallel-group, dose-ranging, phase 2 trial. Lancet Glob. Health 2017, 5, e688-e698. [CrossRef]

32. Bustinduy, A.L.; Waterhouse, D.; de Sousa-Figueiredo, J.C.; Roberts, S.A.; Atuhaire, A.; Van Dam, G.J.; Corstjens, P.L.; Scott, J.T.; Stanton, M.C.; Kabatereine, N.B.; et al. Population pharmacokinetics and pharmacodynamics of praziquantel in Ugandan children with intestinal schistosomiasis: Higher dosages are required for maximal efficacy. mBio 2016, 7, e00227-16. [CrossRef]

33. White, N.J. Intermittent presumptive treatment for malaria. PLoS Med. 2005, 2, e3. [CrossRef]

34. Meyer, T.; Sekljic, H.; Fuchs, S.; Bothe, H.; Schollmeyer, D.; Miculka, C. Taste, a new incentive to switch to (R)-praziquantel in schistosomiasis treatment. PLoS Negl. Trop. Dis. 2009, 3, e357. [CrossRef]

35. el-Lakkany, N.M.; el-Din, S.H.; Sabra, A.N.; Hammam, O.A. Pharmacodynamics of mefloquine and praziquantel combination therapy in mice harbouring juvenile and adult Schistosoma mansoni. Memorias do Instituto Oswaldo Cruz 2011, 106, 814-822. [CrossRef]

36. Mnkugwe, R.H.; Minzi, O.S.; Kinung'hi, S.M.; Kamuhabwa, A.A.; Aklillu, E. Efficacy and safety of praziquantel for treatment of Schistosoma mansoni infection among school children in Tanzania. Pathogens 2019, 9, 28. [CrossRef]

37. Castro, N.; Medina, R.; Sotelo, J.; Jung, H. Bioavailability of praziquantel increases with concomitant administration of food. Antimicrob. Agents Chemother. 2000, 44, 2903-2904. [CrossRef] [PubMed]

38. Olliaro, P.; Delgado-Romero, P.; Keiser, J. The little we know about the pharmacokinetics and pharmacodynamics of praziquantel (racemate and R-enantiomer). J. Antimicrob. Chemother. 2014, 69, 863-870. [CrossRef] [PubMed]

39. World Health Organization. Guidelines for the Treatment of Malaria, 3rd ed.; World Health Organization: Geneva, Switzerland, 2015; Available online: https://www.who.int/malaria/publications/atoz/9789241549127/en/ (accessed on 6 November 2019).

40. European Medicine Agency. Guideline on Bioanalytical Method Validation EMEA/CHMP/EWP/192217/2009 Rev. 1 Corr. 2. Available online: https://www.ema.europa.eu/en/documents/scientific-guideline/guideline-bioanalytical-method-validation en.pdf (accessed on 10 March 2021).

41. R Core Team. R: A language and Environment for Statistical Computing; R Foundation for Statistical Computing: Vienna, Austria, 2020; Available online: https: / www.R-project.org/ (accessed on 10 February 2021).

42. Denney, W.; Duvvuri, S.; Buckeridge, C. Simple, Automatic Noncompartmental Analysis: The PKNCA R Package. J. Pharmacokinet. Pharmacodyn. 2015, 42, S65. [CrossRef] 